CONFORMAL GEOMETRY AND DYNAMICS

An Electronic Journal of the American Mathematical Society

Volume 11, Pages 101-106 (June 13, 2007)

S $1088-4173(07) 00162-2$

\title{
NORMAL FAMILIES OF HOLOMORPHIC FUNCTIONS WITH MULTIPLE ZEROS
}

\author{
XUECHENG PANG, MINGLIANG FANG, AND LAWRENCE ZALCMAN
}

\begin{abstract}
Let $\mathcal{F}$ be a family of functions holomorphic on a domain $D$ in $\mathbb{C}$, all of whose zeros are multiple. Let $h$ be a function meromorphic on $D$, $h \not \equiv 0, \infty$. Suppose that for each $f \in \mathcal{F}, f^{\prime}(z) \neq h(z)$ for $z \in D$. Then $\mathcal{F}$ is a normal family on $D$.
\end{abstract}

\section{INTRODUCTION}

This paper is a complement to [5], where the following result was established.

Theorem A ([5, Theorem 3]). Let $\mathcal{F}$ be a family of functions meromorphic on a domain $D$ in $\mathbb{C}$, all of whose zeros have multiplicity at least 4 . Let $h$ be a function holomorphic on $D, h \neq \equiv$. Suppose that for each $f \in \mathcal{F}, f^{\prime}(z) \neq h(z)$ for $z \in D$. Then $\mathcal{F}$ is a normal family on $D$.

We show by the following example that the constant 4 in Theorem A is sharp.

Example. Let $D=\{z:|z|<1\}$ and $\mathcal{F}=\left\{f_{n}\right\}$, where

$$
f_{n}(z)=\frac{\left(z-\frac{1}{n}\right)^{3}}{z-\frac{3}{n}}=z^{2}+\frac{3}{n^{2}}+\frac{8}{n^{3}\left(z-\frac{3}{n}\right)} .
$$

Clearly, $\mathcal{F}$ fails to be equicontinuous at 0 and hence is not normal in any neighborhood of 0 . However, all zeros of functions in $\mathcal{F}$ have multiplicity 3 , and $f_{n}^{\prime}(z) \neq 2 z$ on $\mathbb{C}$.

Here we prove the following theorem.

Theorem. Let $\mathcal{F}$ be a family of functions holomorphic on a domain $D$ in $\mathbb{C}$, all of whose zeros are multiple. Let $h$ be a function meromorphic on $D, h \neq 0, \infty$. Suppose that for each $f \in \mathcal{F}, f^{\prime}(z) \neq h(z)$ for $z \in D$. Then $\mathcal{F}$ is a normal family on $D$.

Easy examples show that no such result holds if the assumption of multiple zeros is dropped.

The proof of the Theorem follows the proof of Theorem 2 in [5] rather closely. However, certain new difficulties already arise in the case $h(z)=z$. The reader is

Received by the editors February 28, 2007.

2000 Mathematics Subject Classification. Primary 30D45.

The first author's research was supported by the NNSF of China (Grant No. 10671067).

The second author's research was supported by the NNSF of China (Grant No. 10471065).

The third author's research was supported by the German-Israeli Foundation for Scientific Research and Development, Grant G-809-234.6/2003.

(C)2007 American Mathematical Society Reverts to public domain 28 years from publication 
invited to try to adapt the proof of [5] to this special case before reading the proof given below.

\section{Auxiliary Results}

First let us set some notation. We denote the unit disc by $\Delta$. More generally, $\Delta(w, r)=\{z:|z-w|<r\}$ and $\Delta^{\prime}(w, r)=\{z: 0<|z-w|<r\}$.

Our theorem is a generalization of the following result, which is a special case of [6. Theorem 1.1] (cf. [2, Lemma 6] and [5, Theorem 1]).

Lemma 1. Let $\mathcal{F}$ be a family of functions holomorphic on a domain $D$ in $\mathbb{C}$, all of whose zeros are multiple. Let $h$ be a function meromorphic on $D, h \neq \equiv \infty$, such that $h(z) \neq 0$ for $z \in D$. Suppose that for each $f \in \mathcal{F}, f^{\prime}(z) \neq h(z)$ for $z \in D$. Then $\mathcal{F}$ is a normal family on $D$.

Let $g$ be meromorphic on the domain $D$ in $\mathbb{C}$. Then

$$
g^{\#}(z)=\frac{\left|g^{\prime}(z)\right|}{1+|g(z)|^{2}}
$$

is the spherical derivative of $g$. It is easy to see that a meromorphic function with bounded spherical derivative can have order at most 2. For entire functions, more can be said.

Lemma 2 ([1, Theorem 3], cf. [3, Theorem 5]). An entire function which has bounded spherical derivative is of exponential type.

We also require the following renormalization result, which has become a standard tool in the study of normal families.

Lemma 3 ([4, Lemma 2], cf. [7, pp. 216-217]). Let $\mathcal{F}$ be a family of functions meromorphic on the unit disc, all of whose zeros have multiplicity of at least $k$, and suppose that there exists $A \geq 1$ such that $\left|f^{(k)}(z)\right| \leq A$ whenever $f(z)=0$. Then if $\mathcal{F}$ is not normal, there exist, for each $0 \leq \alpha \leq k$,

(a) a number $0<r<1$,

(b) points $z_{n}, \quad\left|z_{n}\right|<r$,

(c) functions $f_{n} \in \mathcal{F}$, and

(d) positive numbers $\rho_{n} \rightarrow 0$

such that $\rho_{n}^{-\alpha} f_{n}\left(z_{n}+\rho_{n} \zeta\right)=g_{n}(\zeta) \rightarrow g(\zeta)$ locally uniformly with respect to the spherical metric, where $g$ is a nonconstant meromorphic function on $\mathbb{C}$ such that $g^{\#}(\zeta) \leq g^{\#}(0)=k A+1$.

Naturally, when the functions in Lemma 3 are holomorphic, convergence is locally uniform (with respect to the Euclidean metric) and the limit function is entire.

Finally, a simple application of Lemma 3 yields the following result.

Lemma 4. Let $\left\{f_{n}\right\}$ be a sequence of functions holomorphic on a domain $D$ in $\mathbb{C}$, all of whose zeros are multiple. Let $\left\{h_{n}\right\}$ be a sequence of functions holomorphic on $D$ such that $h_{n} \rightarrow h$ locally uniformly on $D$, where $h(z) \neq 0$ for $z \in D$. Suppose that for each $n, f_{n}^{\prime}(z) \neq h_{n}(z)$ for $z \in D$. Then $\left\{f_{n}\right\}$ is a normal family on $D$.

Proof. Otherwise, there is a disc (which we may assume to be $\Delta$ ) contained in $D$ on which $\left\{f_{n}\right\}$ is not normal. Then, taking an appropriate subsequence of $\left\{f_{n}\right\}$ and 
renumbering, we have, by Lemma 3 (with $\alpha=k=A=1$ ), points $z_{n}\left(\left|z_{n}\right|<r<1\right.$ ) and numbers $\rho_{n} \rightarrow 0^{+}$such that

$$
\frac{f_{n}\left(z_{n}+\rho_{n} \zeta\right)}{\rho_{n}}=g_{n}(\zeta) \rightarrow g(\zeta)
$$

locally uniformly on $\mathbb{C}$, where $g$ is a nonconstant entire function, all of whose zeros are clearly multiple, satisfying $g^{\#}(\zeta) \leq g^{\#}(0)=2$. By Lemma 2 is of exponential type. Taking an additional subsequence if necessary and renumbering, we may assume that $z_{n} \rightarrow z_{0} \in \Delta$, so that $h_{n}\left(z_{n}+\rho_{n} \zeta\right) \rightarrow h\left(z_{0}\right) \neq 0$ locally uniformly on $\mathbb{C}$. Now since $g_{n}^{\prime}(\zeta)=f_{n}^{\prime}\left(z_{n}+\rho_{n} \zeta\right) \neq h_{n}\left(z_{n}+\rho_{n} \zeta\right) \rightarrow h\left(z_{0}\right)$ and $g_{n}^{\prime} \rightarrow g^{\prime}$ locally uniformly on $\mathbb{C}$, it follows from Hurwitz' Theorem that either $g^{\prime}(\zeta) \equiv h\left(z_{0}\right)$ or $g^{\prime}(\zeta) \neq h\left(z_{0}\right)$ for all $\zeta \in \mathbb{C}$. In the first instance, $g(\zeta)=h\left(z_{0}\right) \zeta+C$; in the second, either $g(\zeta)=A e^{B \zeta}+h\left(z_{0}\right) \zeta+C$ or $g(\zeta)=\left(A+h\left(z_{0}\right)\right) \zeta+C$, where $A \neq 0, B \neq 0$, and $C$ are complex constants. In either case, we obtain a contradiction to the fact that all zeros of the nonconstant function $g$ are multiple.

\section{Proof of the Theorem}

By Lemma 1, it suffices to prove that $\mathcal{F}$ is normal at points for which $h(z)=0$. So we may assume, making standard normalizations, that $\mathcal{F}$ satisfies the conditions of the Theorem and that $h$ is holomorphic on $\Delta$, with

$$
h(z)=z^{k}+a_{k+1} z^{k+1}+\cdots=z^{k} b(z), \quad z \in \Delta,
$$

where $k \geq 1, b(0)=1$, and $h(z) \neq 0$ for $0<|z|<1$. The claim is that $\mathcal{F}$ is normal at 0 . Arguing by contradiction, suppose that $\left\{f_{n}\right\} \subset \mathcal{F}$ but no subsequence of $\left\{f_{n}\right\}$ is normal at 0 . Let $\mathcal{F}_{1}=\left\{F_{n}\right\}$, where $F_{n}=f_{n} / h$. If $f \in \mathcal{F}, f^{\prime}(0) \neq h(0)=0$; hence, since all zeros of $f$ are multiple, $f(0) \neq 0$. Thus, for any $F \in \mathcal{F}_{1}, F(0)=$ $f(0) / h(0)=\infty$.

We shall first prove that $\mathcal{F}_{1}$ is normal on $\Delta$. Suppose not. Then, renumbering, we have by Lemma 3 (again with $\alpha=k=A=1$ ) that there exist $F_{n} \in \mathcal{F}_{1}, z_{n} \in \Delta$ $\left(\left|z_{n}\right| \leq r<1\right)$, and $\rho_{n} \rightarrow 0^{+}$such that

$$
\frac{F_{n}\left(z_{n}+\rho_{n} \zeta\right)}{\rho_{n}}=g_{n}(\zeta) \rightarrow g(\zeta)
$$

spherically uniformly on compact subsets of $\mathbb{C}$, where $g$ is a nonconstant meromorphic function on the plane, all of whose zeros are multiple, such that $g^{\#}(\zeta) \leq$ $g^{\#}(0)=2$.

We consider the following two cases.

(a) Suppose $z_{n} / \rho_{n} \rightarrow \infty$. Then, since $g_{n}\left(-z_{n} / \rho_{n}\right)=F_{n}(0) / \rho_{n}$, the pole of $g_{n}$ corresponding to that of $F_{n}$ at 0 drifts off to infinity; $g_{n}$ converges to $g$ locally uniformly on $\mathbb{C}$; and hence, by Lemma $2, g$ is entire of exponential type. We have

$$
F_{n}^{\prime}(z)=\frac{f_{n}^{\prime}(z) h(z)-f_{n}(z) h^{\prime}(z)}{h(z)^{2}}=\frac{f_{n}^{\prime}(z)}{h(z)}-\frac{h^{\prime}(z)}{h(z)} F_{n}(z) .
$$

Thus,

$$
\begin{aligned}
g_{n}^{\prime}(\zeta) & =F_{n}^{\prime}\left(z_{n}+\rho_{n} \zeta\right)=\frac{f_{n}^{\prime}\left(z_{n}+\rho_{n} \zeta\right)}{h\left(z_{n}+\rho_{n} \zeta\right)}-\frac{h^{\prime}\left(z_{n}+\rho_{n} \zeta\right)}{h\left(z_{n}+\rho_{n} \zeta\right)} F_{n}\left(z_{n}+\rho_{n} \zeta\right) \\
& =\frac{f_{n}^{\prime}\left(z_{n}+\rho_{n} \zeta\right)}{h\left(z_{n}+\rho_{n} \zeta\right)}-\left(\frac{k}{z_{n} / \rho_{n}+\zeta}+\rho_{n} \frac{b^{\prime}\left(z_{n}+\rho_{n} \zeta\right)}{b\left(z_{n}+\rho_{n} \zeta\right)}\right) \frac{F_{n}\left(z_{n}+\rho_{n} \zeta\right)}{\rho_{n}}
\end{aligned}
$$


Clearly,

$$
\lim _{n \rightarrow \infty} \frac{k}{z_{n} / \rho_{n}+\zeta}=0 \quad \text { and } \quad \lim _{n \rightarrow \infty} \rho_{n} \frac{b^{\prime}\left(z_{n}+\rho_{n} \zeta\right)}{b\left(z_{n}+\rho_{n} \zeta\right)}=0
$$

uniformly on compact sets of $\mathbb{C}$. Thus,

$$
\frac{f_{n}^{\prime}\left(z_{n}+\rho_{n} \zeta\right)}{h\left(z_{n}+\rho_{n} \zeta\right)}=g_{n}^{\prime}(\zeta)+\left(\frac{k}{z_{n} / \rho_{n}+\zeta}+\rho_{n} \frac{b^{\prime}\left(z_{n}+\rho_{n} \zeta\right)}{b\left(z_{n}+\rho_{n} \zeta\right)}\right) g_{n}(\zeta)
$$

converges locally uniformly to $g^{\prime}(\zeta)$ on $\mathbb{C}$. Since $f_{n}^{\prime}(z) / h(z) \neq 1$, by Hurwitz' Theorem either $g^{\prime} \equiv 1$ or $g^{\prime}(\zeta) \neq 1$ for all $\zeta \in \mathbb{C}$. Just as in the proof of Lemma 4, neither of these alternatives is consistent with the fact that all zeros of $g$ are multiple.

(b) So we may assume that $z_{n} / \rho_{n} \rightarrow \alpha$, a finite complex number. We have

$$
\frac{F_{n}\left(\rho_{n} \zeta\right)}{\rho_{n}}=\frac{F_{n}\left(z_{n}+\rho_{n}\left(\zeta-z_{n} / \rho_{n}\right)\right)}{\rho_{n}} \rightarrow g(\zeta-\alpha)=\tilde{g}(\zeta)
$$

the convergence being spherically uniform on compact sets of $\mathbb{C}$ and hence uniform on compacta disjoint from the poles of $\tilde{g}$. Clearly, all zeros of $\tilde{g}$ are multiple and $\tilde{g}$ has a single pole at 0 , of order $k$.

Now

$$
\lim _{n \rightarrow \infty} \frac{h\left(\rho_{n} \zeta\right)}{\rho_{n}^{k}}=\zeta^{k}
$$

uniformly on compact subsets of $\mathbb{C}$. Thus, writing

$$
G_{n}(\zeta)=\frac{f_{n}\left(\rho_{n} \zeta\right)}{\rho_{n}^{k+1}}=\frac{h\left(\rho_{n} \zeta\right)}{\rho_{n}^{k}} \frac{f_{n}\left(\rho_{n} \zeta\right)}{\rho_{n} h\left(\rho_{n} \zeta\right)}=\frac{h\left(\rho_{n} \zeta\right)}{\rho_{n}^{k}} \frac{F_{n}\left(\rho_{n} \zeta\right)}{\rho_{n}},
$$

we have

$$
G_{n}(\zeta) \rightarrow \zeta^{k} \tilde{g}(\zeta)=G(\zeta)
$$

locally uniformly on $\mathbb{C} \backslash\{0\}$ and hence (by the maximum principle) locally uniformly on $\mathbb{C}$. Clearly, $G$ is an entire function, all of whose zeros are multiple, which has bounded spherical derivative and hence is of exponential type. Since the pole of $\tilde{g}$ at 0 has order $k, G(0) \neq 0$.

We claim that $G^{\prime}(\zeta) \neq \zeta^{k}$ for all $\zeta \in \mathbb{C}$. Indeed, suppose that $G^{\prime}\left(\zeta_{0}\right)=\zeta_{0}^{k}$. Since $G$ is holomorphic and

$$
G_{n}^{\prime}(\zeta)-\frac{h\left(\rho_{n} \zeta\right)}{\rho_{n}^{k}}=\frac{f_{n}^{\prime}\left(\rho_{n} \zeta\right)-h\left(\rho_{n} \zeta\right)}{\rho_{n}^{k}} \neq 0,
$$

we have by Hurwitz' Theorem $G^{\prime}(\zeta) \equiv \zeta^{k}$; hence, $G(\zeta)=\zeta^{k+1} /(k+1)+C$. Since all zeros of $G$ are multiple, $C=0$; but this contradicts $G(0) \neq 0$.

Thus $G^{\prime}(\zeta) \neq \zeta^{k}$ for all $\zeta \in \mathbb{C}$. It follows that either $G(\zeta)=A e^{B \zeta}+\zeta^{k+1} /(k+1)$ $+C$ or $G(\zeta)=\zeta^{k+1} /(k+1)+A \zeta+C$ for complex constants $A \neq 0, B \neq 0$, and $C$. This is inconsistent with the fact that all zeros of $G$ are multiple unless $k=1$ and $G(\zeta)=(\zeta+A)^{2} / 2$. It remains to rule out this last possibility.

Suppose, therefore, that $G(\zeta)=(\zeta+A)^{2} / 2$. Then since

$$
\frac{f_{n}\left(\rho_{n} \zeta\right)}{\rho_{n}^{2}} \rightarrow \frac{(\zeta+A)^{2}}{2}
$$

locally uniformly on $\mathbb{C}$, there exist points $\zeta_{n} \rightarrow-A$ such that $f_{n}\left(\rho_{n} \zeta_{n}\right)=0$. The sequence $\left\{f_{n}\right\}$ fails to be normal at 0 ; on the other hand, by Lemma 1 , $\left\{f_{n}\right\}$ is normal on $\Delta^{\prime}=\{z: 0<|z|<1\}$. Since the functions $f_{n}$ are holomorphic on $\Delta$, it follows that they tend to $\infty$ locally uniformly on $\Delta^{\prime}$. Suppose now that there exists 
$\delta>0$ such that, for each $n, f_{n}$ has only the single zero $\xi_{n}=\rho_{n} \zeta_{n}$ in $\Delta(0, \delta)$ for all $n$. Put

$$
H_{n}(z)=\frac{f_{n}(z)}{\left(z-\xi_{n}\right)^{2}}
$$

Then $\left\{H_{n}\right\}$ is a sequence of nonvanishing holomorphic functions on $\Delta(0, \delta)$ tending to $\infty$ locally uniformly on $\Delta^{\prime}(0, \delta)$. It follows that the sequence $\left\{1 / H_{n}\right\}$ of holomorphic functions tends to 0 locally uniformly on $\Delta^{\prime}(0, \delta)$ and hence, by the maximum principle, on $\Delta(0, \delta)$. Thus $H_{n} \rightarrow \infty$ locally uniformly on $\Delta(0, \delta)$. In particular, $H_{n}\left(2 \rho_{n} \zeta_{n}\right) \rightarrow \infty$. But by (1) and (2),

$$
H_{n}\left(2 \rho_{n} \zeta_{n}\right)=\frac{f_{n}\left(2 \rho_{n} \zeta_{n}\right)}{\left(\rho_{n} \zeta_{n}\right)^{2}} \rightarrow \frac{1}{2}
$$

a contradiction.

Thus, taking a subsequence if necessary, we may assume that for any $\delta>0, f_{n}$ has at least two distinct zeros in $\Delta(0, \delta)$ for $n$ sufficiently large. Choose $\eta_{n}$ such that $f_{n}\left(\eta_{n}\right)=0$ and $f_{n}$ has no zeros on $\Delta^{\prime}\left(\xi_{n},\left|\eta_{n}-\xi_{n}\right|\right)$; then $\eta_{n} \rightarrow 0$. By (1), $\eta_{n} / \rho_{n} \rightarrow \infty$, so that $\xi_{n} / \eta_{n}=\rho_{n} \zeta_{n} / \eta_{n} \rightarrow 0$. Put

$$
K_{n}(z)=\frac{f_{n}\left(\left(\eta_{n}-\xi_{n}\right) z\right)}{\left(\eta_{n}-\xi_{n}\right)^{2}}, \quad h_{n}(z)=\frac{h\left(\left(\eta_{n}-\xi_{n}\right) z\right)}{\eta_{n}-\xi_{n}} .
$$

Then $\left\{K_{n}\right\}$ is a sequence of holomorphic functions, all of whose zeros are multiple, which are defined for each $z \in \mathbb{C}$ for $n$ sufficiently large. Similarly, the sequence of holomorphic functions $\left\{h_{n}\right\}$ is defined for each $z \in \mathbb{C}$ for $n$ sufficiently large; and $h_{n}(z) \rightarrow z$ locally uniformly on $\mathbb{C}$. Clearly, $K_{n}^{\prime}(z) \neq h_{n}(z)$. Hence, by Lemma $4\left\{K_{n}\right\}$ is normal on $\mathbb{C} \backslash\{0\}$. We claim that $\left\{K_{n}\right\}$ is also normal at 0 . Indeed, otherwise $K_{n} \rightarrow \infty$ locally uniformly on $\mathbb{C} \backslash\{0\}$. But this is impossible, as $K_{n}\left(\eta_{n} /\left(\eta_{n}-\xi_{n}\right)\right)=0$ and $\eta_{n} /\left(\eta_{n}-\xi_{n}\right) \rightarrow 1$. Thus $\left\{K_{n}\right\}$ is normal on $\mathbb{C}$. Taking a subsequence and renumbering, we have $K_{n} \rightarrow K$ locally uniformly on $\mathbb{C}$ for an entire function $K$, all of whose zeros are multiple. Since $K_{n}^{\prime}(z) \neq h_{n}(z)$ and $h_{n}(z) \rightarrow z$, either $K^{\prime}(z) \neq z$ for all $z \in \mathbb{C}$ or $K^{\prime}(z) \equiv z$. But $K_{n}\left(\xi_{n} /\left(\eta_{n}-\xi_{n}\right)\right)=0$ and $\xi_{n} /\left(\eta_{n}-\xi_{n}\right) \rightarrow 0$, so that $K(0)=0$ and hence $K^{\prime}(0)=0$. Thus $K^{\prime}(z) \equiv z$. It follows that $K(z) \equiv z^{2} / 2$. But $K_{n}\left(\eta_{n} /\left(\eta_{n}-\xi_{n}\right)\right)=0$ and $\eta_{n} /\left(\eta_{n}-\xi_{n}\right) \rightarrow 1$, so that $K(1)=0$. This contradiction completes the proof that $\mathcal{F}_{1}$ is normal on $\Delta$.

Finally, since $\mathcal{F}_{1}$ is normal on $\Delta$ and $F_{n}(0)=\infty$ for all $n$, there exists $\delta>0$ such that $\left|F_{n}(z)\right| \geq 1$ on $\Delta(0, \delta)$ for all $n$. Thus $f_{n}(z) \neq 0$ on $\Delta(0, \delta)$, and hence $1 / f_{n}$ is holomorphic on $\Delta(0, \delta)$ for all $n$. Choosing $\delta$ small enough that $|h(z)| \geq|z|^{k} / 2$ for $|z| \leq \delta$, we have

$$
\left|\frac{1}{f_{n}(z)}\right|=\left|\frac{1}{F_{n}(z)} \cdot \frac{1}{h(z)}\right| \leq \frac{2^{k+1}}{\delta^{k}}, \quad|z|=\frac{\delta}{2} .
$$

By the maximum principle, this bound holds throughout $\Delta(0, \delta / 2)$. It follows that $\left\{f_{n}\right\}$ is normal at 0 , which contradicts the original choice of $\left\{f_{n}\right\}$. Thus $\mathcal{F}$ is normal at 0 , and the Theorem is proved.

\section{REFERENCES}

[1] J. Clunie and W. K. Hayman, The spherical derivative of integral and meromorphic functions, Comment. Math. Helv. 40 (1966), 117-148. MR0192055 (33:282)

[2] Ming-liang Fang, A note on a problem of Hayman, Analysis 20 (2000), 45-49. MR:1757068 (2001a:30036) 
[3] David Minda, Yosida functions, Lectures on Complex Analysis (Xian, 1987), (Chi Tai Chuang, ed.), World Scientific Pub. Co., Singapore, 1988, pp. 197-213. MR996476 (90d:30097)

[4] Xuecheng Pang and Lawrence Zalcman, Normal families and shared values, Bull. London Math. Soc. 32 (2000), 325-331. MR 1750485 (2001e:30059)

[5] Xuecheng Pang and Lawrence Zalcman, Normal families of meromorphic functions with multiple zeros and poles, Israel J. Math. 136 (2003), 1-9. MR.1998102 (2004f:30025)

[6] Xuecheng Pang, Degui Yang, and Lawrence Zalcman, Normal families and omitted functions, Indiana Univ. Math. J. 54 (2005), 223-235. MR.2126722 (2005j:30045)

[7] Lawrence Zalcman, Normal families: new perspectives, Bull. Amer. Math. Soc. (N.S.) 35 (1998), 215-230. MR1624862 (99g:30048)

Department of Mathematics, East China Normal University, Shanghai 200062, PeoPLE's RePublic of China

E-mail address: xcpang@euler.math.ecnu.edu.cn

Institute of Applied Mathematics, South China Agricultural University, Guangzhou 510642, People's Republic of China

E-mail address: hnmlfang@hotmail.com

Department of Mathematics, Bar-Ilan University, 52900 Ramat-Gan, Israel

E-mail address: zalcman@macs.biu.ac.il 